\title{
Suppression of flow separation of power-law fluids flow around a confined circular cylinder by superimposed thermal buoyancy
}

\author{
H. Laidoudi*, M. Bouzit** \\ *University of Sciences and Technology of Oran, BP 1505, El-Menaouer, Oran 3100, Algeria, \\ E-mail: hichemsot19@Gmail.com \\ **University of Sciences and Technology of Oran, BP 1505, El -Menaouer, Oran 3100, Algeria, \\ E-mail: Bouzit_mohamed@yahoo.fr
}

cross $^{\text {ref }}$ http://dx.doi.org/10.5755/j01.mech.23.2.14342

\section{Nomenclature}

$B$ - volume expansion coefficient, $1 / \mathrm{K} ; C_{f}$ - skin friction coefficient; $C_{D}$-drag coefficient; $d$-cylinder size, $\mathrm{m}$; $g$ - gravitational acceleration, $\mathrm{m} / \mathrm{s}^{2} ; G r$ - Grashof number; $H$ - Channel width, $\mathrm{m} ; h$ - Local convective heat transfer coefficient, $\mathrm{W} /\left(\mathrm{m}^{2} \mathrm{~K}\right) ; I_{2}$ - second invariant of the rate of deformation tensor, $\mathrm{s}^{2} ; k$-fluid thermal conductivity, $\mathrm{W} /\left(\mathrm{m}^{2} \mathrm{~K}\right) ; \quad L_{r}$-dimensionless recirculation length; $L_{r}^{*}$ - recirculation length, $\mathrm{m} ; L_{d}$-downstream distance, $\mathrm{m}$; $L_{u}$ - upstream distance, $\mathrm{m} ; m$-power-law consistency index, $\quad \mathrm{Pa} \mathrm{s}^{\mathrm{n}} ; \quad n$-power-law flow behavior index; $n_{s}$ - direction normal to the cylinder surface; $\mathrm{Nu}$ - average total Nusselt number; $p$ - pressure, $\mathrm{Pa} ; P$ - dimensionless pressure; $P r$-Prandtl number; $P e$-Peclet number; $R e$ - Reynolds number; $R i$-Richardson number; $T$ - temperature, $\mathrm{K} ; \quad U$-dimensionless cross-stream velocity; $u$ - cross-stream velocity, $\mathrm{m} / \mathrm{s} ; V$-dimensionless stream-wise velocity; $v$-velocity stream-wise velocity, $\mathrm{m} / \mathrm{s} ; \quad X$-dimensionless cross-stream coordinate; $Y$ - dimensionless stream-wise coordinate; $y$-stream-wise coordinate, $\mathrm{m}$;

greek letters -

$\varepsilon$-rate of deformation, $\mathrm{s}^{-1} ; \eta$-viscosity, Pa s; $\rho$ - fluid density $\mathrm{kg} / \mathrm{m}^{3} ; \beta$-blockage ratio; $\tau$-shear stress rate tensor, $\mathrm{Pa} ; \theta$ - dimensionless temperature;

subscript -

in - inlet; out-outlet; $\quad w$-wall; $\max$-maximum; ave - average; $c$ - critical.

\section{Introduction}

The heat transfer driven by buoyancy force aspects of a confined obstacle cylinder is a fundamental problem in fluid mechanics. This type of flow can be used as a key issue in design and development of such products [1], or can be used as a validation in academic researches [2]. The separation flow behind an obstacle is an undesirable and detrimental flow phenomenon in many engineering applications. The separation mainly causes increase of hydrodynamics forces (drag, lift) [3], loss flowing power which pose serious challenges practical engineering application [4]. For the completed application range, experimental and numerical research have indicated that the generation of flow separation depends on its flow conditions as well as geometrical parameters (crosssectional shape, channels) [5-10]. In those literatures there are some detailed information exists on the flow and heat transfer around circular [5], square [6], and other crosssectional shape (semi circular, triangular). Recently more attention is conducted to control the flow separation. Several aspects have so far been established to control the boundary layer separation. There are some earlier information excite on the control of flow $[11,12]$. In those literatures, the laminar flow is always at a high risk of boundary layer separation since the laminar boundary layer can support only a very small adverse pressure gradient without the occurrence of separation. On the other hand, the danger of separation is intrinsically reduced in case of the turbulent flow because of the continuous supply of momentum from the external flow towards the wall due to the turbulent mixing. Dipankar Chatterjee et al. [13] numerically studied the phenomena of suppression of flow separation around bluff obstacles by superimposed thermal buoyancy. They have considered an unconfined flow of a Newtonian fluid around heated cylinders of circular and square cross-sections in a buoyancy-aided vertical flow configuration; The Reynolds numbers are limited between $10-40$. The results showed that, when the buoyancy is added, the flow separation diminishes gradually and at some value of the buoyancy parameter it completely disappears. They have added also, the effects of Richardson number on a rate of heat transfer.

The majority of the researches reported in this field (control of flow separation, and mixed convection) deals with the flow of Newtonian fluids past around cylinder. In contrast little information is available on the suppression of Non-Newtonian fluid flow separation around a confined square cylinder by superimposed thermal buoyancy.

Indeed, many common substances exhibit NonNewtonian flows; these include polymeric, cosmetics and toothpaste, natural substances, biological fluids, foods, etc. Theoretically, Non-Newtonian power-law fluid is type of generalized Newtonian fluid for which the apparent viscosity characteristic depends with shear rate. The most common type of Non-Newtonian power law behavior is shear thickening fluid $(n>1)$, where the viscosity appears to increase when the shear rate increases [14]. Another familiar example of the opposite. The shear thinning fluid $(n<1)$ [14], where the viscosity appears to decrease when the shear rate increases. These two behaviors may be represented by the power-law model.

Furthermore, thermal buoyancy is believed to play an important role on the wake behind a confined obstacle cylinder. The principal parameter that controls a relative effect of buoyancy is the Richardson number $(R i)$, 
defined as $\left(R i=G r / R e^{2}\right)$, where, $G r$ is Grashof number, and $R e$ is Reynolds number. This number determines the relative importance of forced and natural convection. The free convection dominates over the forced convection when $R i>1$, and the forced convection dominates when $R i<1$. Both the free and forced convection dominate equally when $R i$ is nearly approached to 1 and the resulting thermal transport becomes mixed convection dominated.

There have been some researches in the flied of flow pasts around a cylinder for the sake of suppression the flow separation behind obstacles by playing on the crosssectional shape of the obstacle or the flow condition. For example, Dipankar Chatterjee et al. [15] they presented the effects of thermal buoyancy and Prandtl number on Newtonian fluid flow characteristics and mixed convection heat transfer over two equal isothermal square cylinders placed in a tandem arrangement within a horizontal channel at low Reynolds numbers. The results showed that, in all cases the recirculation length of the upstream cylinder is larger than the corresponding value for the downstream cylinder for the range of Reynolds numbers considered in this study $1-30$. Furthermore, with higher Richardson number the length of the recirculation zone increases. Jaber Aboueian, et el. [16] they studied the effect of inclination angle on the steady flow and forced convection of power-law fluids around a heated inclined square cylinder in an horizontal channel, the numerical results are presented and discussed under these condition : $(0.4 \leq n \leq 1.6),(0 \leq \alpha \leq 48), \beta=0.25$ and $(1 \leq \operatorname{Re} \leq 40)$, $\operatorname{Pr}=50$, they found that at $\operatorname{Re}=10$ there is no flow separation on the square cylinder in $\alpha=45$ for all powerlaw indices and in $\alpha=15$ and 30 for $n=0.4$. They also found that the onset of wake formation is delayed with an increase in the inclination angle, the length of the recirculation zone increases with an increase in Reynolds number and decreases with an increase in power-law index for all inclination angles at least for $R e$ 20-40. E. Nikfarjam, Sohankar A. [17] Numerically investigated the free-stream flow of power-law fluids and forced convection heat transfer around a square cylinder and two square cylinders in a tandem arrangement by solving continuity, momentum and energy equations in 2-D directions. In the single cylinder case, the power-law index and Reynolds numbers range from $n=0.7$ to 1.4 and $R e=60$ to 160 at $P r=0.7$. The results showed that, the mean wake length decreases dramatically as the Reynolds number increases. For a fixed value of Reynolds number, the mean wake length increases gradually with the powerlaw index. The recent trend is completely inversed when the blockage ration increases to the value 0.25 [16]. Moreover [18] studied numerically the effect of vortex shedding from square cylinder under aiding thermal buoyancy by solving continuity, momentum, and energy equations.

Based on the mentioned reviews. In this study, our objective is to analyze through numerical simulation the phenomena of suppression of Non- Newtonian powerLaw fluid flow separation around a confined circular cylinder by superimposed thermal buoyancy in a vertical confined channel, and exploring the effects thermal buoyancy on the flow. In particular, numerical results are presented and discussed for the following conditions: $(0.4 \leq n \leq 1.2), \beta=0.20$ and $(10 \leq R e \leq 40),(0 \leq R i \leq 0.8)$, $\operatorname{Pr}=50$. However, this work addresses the combined characteristics of power low fluid flow and mixed convection, Also larges the previous research in this field, in fact this research improves heat $\mathrm{Nu}$ effects insights and gives the exact critical quantitative of buoyancy strength on wake behind the confined cylinder.

\section{Problem statement and governing equation}

The problem under consideration is shown schematically in Fig. 1 we consider a heated circular cylinder, is located in the cross middle of a long twodimensional vertical channel. The present research aims to investigate numerically the incompressible flow of powerlaw fluids around this obstacle. Due to numerical considerations, the fluids flow enters the channel with fully developed velocity $\left(V_{\max }\right)$ profile and constant temperature $\left(T_{i n}\right)$, and passes the cylinder, whose surface is maintained at constant temperature $\left(T_{w}\right)$. the length of confined cylinder is defined $(d)$, the ratio of this length to the height of the channel $(H)$, defines the blockage ratio $(\beta=0.2)$, The distance between the center of the cylinder and the channel inlet $\left(L_{u}\right)$ is 10 times of the cylinder height, the distance between the center of the cylinder and the channel outlet $\left(L_{d}\right)$ is 20 times of the cylinder height. The flow and heat transfer phenomena are governed by continuity, momentum and energy equations.

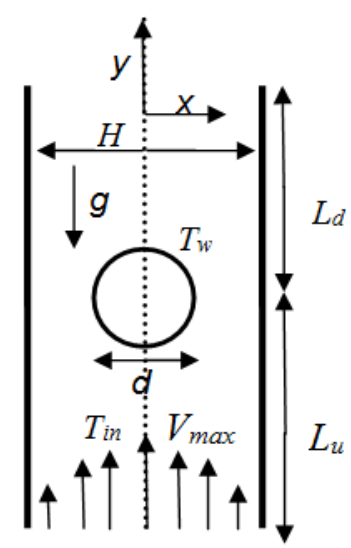

Fig. 1 Schematic diagram of the computational domain

The governing equations subjected to the Boussinesq approximation and negligible dissipation effects are written in their dimensionless forms as follows:

- continuity:

$\frac{\partial U}{\partial X}+\frac{\partial V}{\partial Y}=0$

- momentum:

$$
\begin{aligned}
& U \frac{\partial U}{\partial X}+V \frac{\partial U}{\partial Y}=-\frac{\partial P}{\partial X}+\frac{1}{\operatorname{Re}}\left(\frac{\partial^{2} U}{\partial X^{2}}+\frac{\partial^{2} V}{\partial Y^{2}}\right) \\
& U \frac{\partial V}{\partial X}+V \frac{\partial V}{\partial Y}=-\frac{\partial P}{\partial Y}+\frac{1}{\operatorname{Re}}\left(\frac{\partial^{2} U}{\partial X^{2}}+\frac{\partial^{2} V}{\partial Y^{2}}\right)+\operatorname{Ri\theta }
\end{aligned}
$$

- thermal energy:

$$
U \frac{\partial \theta}{\partial X}+V \frac{\partial \theta}{\partial Y}=+\frac{1}{P e}\left(\frac{\partial^{2} \theta}{\partial X^{2}}+\frac{\partial^{2} \theta}{\partial Y^{2}}\right)
$$




$$
\begin{aligned}
& U=\frac{u}{V_{\text {max }}} ; V=\frac{v}{V_{\text {max }}} ; X=\frac{x}{d} ; Y=\frac{y}{d} ; \\
& P=\frac{p}{\rho V_{\text {max }}^{2}} ; \theta=\frac{T-T_{i n}}{T_{w}-T_{i n}},
\end{aligned}
$$

where $U$ and $V$ are the fluid dimensionless velocities in $X$ and $Y$ directions; $P$ and $\theta$ are dimensionless pressure and temperature respectively and $R e$ and $P e$ is Reynolds and Peclet numbers; $R i$ is the Richardson number. The thermophysical properties of the fluid $(\rho, C p, k)$ are considered to be temperature constant, and the contribution of viscous dissipation in the thermal energy equation is also neglected in this work.

The behavior of the power-law fluid is represented by the following equation:

$$
\tau_{i j}=2 \eta \varepsilon_{i j},
$$

where $\tau_{i j}$ and $\varepsilon_{i j}$ are the rate of deformation and viscous stress tensors, respectively. In addition, $\eta$ which represents the fluid viscosity, is defined (in dimensional form) as follows for power-law fluids:

$$
\eta=m\left(\frac{I_{2}}{2}\right)^{\left(\frac{n-1}{2}\right)},
$$

where $n$ is the power-law index; $m$ is the consistency index and $I_{2}$ is the second invariant of the rate of deformation tensor. In the Cartesian coordinates, this invariant is computed by the following equation:

$$
\frac{I_{2}}{2}=2\left(\frac{\partial U}{\partial X}\right)^{2}+2\left(\frac{\partial V}{\partial Y}\right)^{2}+\left(\frac{\partial U}{\partial X}+\frac{\partial U}{\partial Y}\right)^{2} .
$$

Generally, Reynolds number, Prandtl and Grashof number for power-law fluids are computed as follows:

$$
\begin{aligned}
& R e=\frac{\rho d^{n} V_{\max }^{(n-2)}}{m} ; \operatorname{Pr}=\frac{m c_{p}}{k}\left(\frac{V_{\max }}{d}\right)^{(n-1)} ; \\
& G r=\frac{g B\left(T_{w}-T_{i n}\right) d(2 n+1) V_{\max }^{(2-2 n)}}{m^{2}},
\end{aligned}
$$

where $\rho, c p$ and $k$ are density, specific heat and thermal conductivity of the fluid, respectively, and $B$ are the gravitational acceleration and volumetric expansion coefficient. The Peclet and Richardson numbers that are showed in governing equations are obtained from the following equation:

$$
\begin{aligned}
& P e=\operatorname{Re} \times \operatorname{Pr}=\frac{\rho c_{p} d V_{\max }}{k} ; \\
& R i=\frac{G r}{R e^{2}}=\frac{g B\left(T_{w}-T_{i n}\right) d V_{\max }^{(n-2)}}{k} .
\end{aligned}
$$

The boundary conditions used for the flow and heat configuration are:

At the inlet a fully developed velocity profile forlaminar flow of power-law fluids with a constant temperature, this is given by:

$$
u=0 ; v=V_{\text {max }}(1-|2 X \beta|)^{\left(\frac{n+1}{n}\right)} ; T=T_{\text {in }} .
$$

On the surface of the obstacle cylinder: The standard no-slip condition is used and the cylinder is maintained and heated with a constant temperature $T_{w}$.

$$
u=0 ; v=0 ; T=T_{w} .
$$

At the channel walls, the usual no-slip condition for flow and adiabatic condition for energy are used.

$$
u=0 ; v=0 ; \text { Adiabatic . }
$$

At the outlet Neumann boundary condition for field variables is employed:

$$
\frac{\partial U}{\partial X}=0 ; \frac{\partial V}{\partial Y}=0 ; \frac{\partial \theta}{\partial Y}=0
$$

\section{Numerical methodology}

The conservation equations subjected to the aforementioned boundary conditions are solved using a finite volume based CFD solver ANSYS- CFX version (14.0). The ANSYS- CFX software is a high performance, a general purpose fluid dynamics program that is capable of solving diverse and complex three dimensional geometries. This code uses the above equations to describe the principal processes of momentum, mass, and heat transfer; it also combines a specific number of mathematical models such as (power-law, $k-e \ldots$ ) that can be used simultaneously with fundamental equations to describe other physical and chemical phenomena such as combustion, turbulence, Non-Newtonian flow etc. This present CFD package applies the finite volume method to covert the governing partial differential equations into a system of discrete algebraic equations by discretizing the computational domain into grid mesh. These equations may result in a solution with specified domain boundary conditions.

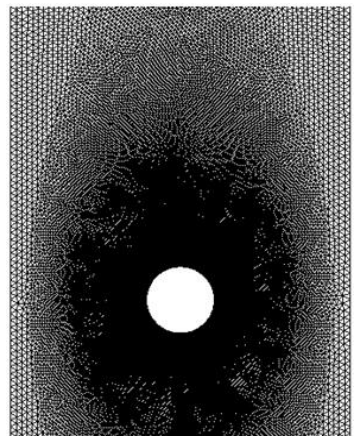

Fig. 2 Typical grids used for simulation

The unstructured trilateral cells of non-uniform grid spacing were generated using the grid package GAMBIT (version 2.4.6). The grids points are distributed in a non-uniform manner wither higher concentration near cylinder Fig. 2 shows the grid used for circular cylinder. Since the overall error CFD computational is mainly a combination of grid density and convergence criteria. For 
this purpose, before verifying our CFD numerical results first we perform a grid study case. In order to investigate the solution grid independency and to choose the passable mesh solution. Grid independence test was carried out with respect to average values of Nusselt numbers and total drag coefficient $C_{D}$ at Reynolds number of 10, power-law index of 0.4 and Richardson number of 0 , three different meshes were generated viz. Mesh1, Mesh2, and Mesh3.

Table 1 summaries the meshes and corresponding numbers of elements used in this work. Hence $C_{N}$ is the number of el elements on face of cylinder. The results of grid independency have been presented in Table 1. It is evident that average Nusselt number shows variation of $0.20 \%$, and $0.19 \%$ whether total drag coefficient have $0.06 \%$ and $0.09 \%$ with meshes Mesh1 and Mesh3. So for the present computations the grid-independent situation was established for Mesh2 which is computationally economical for all different cases studied in this present literature.

Table 1

Control volumes effects on fluid flow and heat transfer parameters $\operatorname{Re}=10, \operatorname{Pr}=50, n=0.4, R i=0$

\begin{tabular}{|l|l|l|l|l|}
\hline Grid & Elements & $C_{N}$ & $N u$ & $C_{D}$ \\
\hline Mesh1 & 89278 & 250 & 9.707 & 3.296 \\
\hline Mesh2 & 120358 & 310 & 9.727 & 3.294 \\
\hline Mesh3 & 168642 & 400 & 9.46 & 3.291 \\
\hline
\end{tabular}

\subsection{Validation of result}

In order to verify our numerical method, we have conducted two sets of comprehensive tests to evaluate the accuracy of our results in comparison with other available numerical investigations in this field. In the first set, a forced convection of Newtonian and Non-Newtonian fluid flows around a confined square cylinder are performed respectively, and the average Nusselt number of cylinders in the range of $0.4 \leq n \leq 1.4, \operatorname{Re} 20,40$ for $\operatorname{Pr}=50$ and $\beta=0.25$ are presented Table 2. A good agreement is seen between the results of present work and the results reported in the literature of Aboueian J. et al. [16]. The maximum deviation of computed data from the results of prior work is about set and $6.34 \%$. The second set of test is a mixed convection and Newtonian fluid flow over two equal isothermal square cylinders placed in a tandem arrangement within a horizontal channel in the range of this condition as, $1 \leq R e \leq 30$, for $\operatorname{Pr}=0.7,10$, and $R i=0.25$. The computed results are presented in Table 3 . Again a good agreement is observed between predictive results and the results of [15], the maximum deviation is about $0.55 \%$.

Comparison of $\mathrm{Nu}$ at different values of $R e$, $n$ for a square cylinder, $\beta=1 / 4, \operatorname{Pr}=50$

\begin{tabular}{|l|l|l|l|}
\hline$R e$ & $n$ & present & Aboueian J. et al [16] \\
\hline \multirow{3}{*}{20} & 0.4 & 10.103 & 10.787 \\
\cline { 2 - 4 } & 1.0 & 9.166 & 9.219 \\
\cline { 2 - 4 } & 1.4 & 8.775 & 8.6 \\
\hline 40 & 0.4 & 12.946 & 13.768 \\
\cline { 2 - 4 } & 1.0 & 12.262 & 12.313 \\
\cline { 2 - 4 } & 1.4 & 11.685 & 11.492 \\
\hline
\end{tabular}

Table 3

Comparison of $\mathrm{Nu}$ at different values of $\mathrm{Re}$, $\operatorname{Pr}$ for a square cylinder, $R i=0.25$

\begin{tabular}{|l|l|l|l|}
\hline $\operatorname{Pr}$ & $R e$ & Present & Dipankar et al [15] \\
\hline \multirow{3}{*}{10} & 1 & 1.79 & 1.8 \\
\cline { 2 - 4 } & 10 & 3.94 & 3.94 \\
\cline { 2 - 4 } & 30 & 6.37 & 6.4 \\
\hline
\end{tabular}

\section{Results and discussion}

In present study, numerical investigations have been carried out for $R e=10,20,30,40$, and for $n=0.4$ 1.2, at fixed values of $\operatorname{Pr}=50$, and $\beta=1 / 5$. In order to looking for the critical Richardson number for the complete suppression of flow separation around a confined cylinder, the buoyancy effect is studied for the Richardson number range $0-0.8$. The wake formation of NonNewtonian fluids, and flow separation behind the cylinder in the absence of superimposed thermal buoyancy are exhibited through the streamline contours shown in Fig. 3 For representative range of the power-law index and for two values of the Reynolds numbers $R e=20$ and 40. The usual steady nature of the flow field is appeared from these contours. A closed steady recirculation region consisting of twin symmetric vortices generates behind the object. In both stream-wise and transverse directions, the wake increases dramatically as the Reynolds number increases. For a fixed value of Reynolds number, the wake also increases gradually with the power-law index, the recent trend is in line with that observed for confined and unconfined cylinders of different cross-sections, e.g., see [6],[16]. Qualitatively, for this particular ratio $(\beta=0.2)$ the effects of power law index on the wake can be rationalized as follows: For the confined cylinder, the effective shearing increases in the gap between the cylinder and wall which leads to the growth of apparent viscosity for a shearthickening fluid with moving away from the obstacle, resulting a viscous layer protects a region behind the cylinder, so the streamlines cannot penetrate easily and the formation of wake is increased, whereas the apparent viscosity is decreased for a shear-thinning fluid and so the wake formation is delayed. Finally, it can be estimated that increase in the value of power-law index and/or Reynolds amplify the hydrodynamic instability of flow.

The steady thermal field under similar conditions is also illustrated in Fig. 3. Since the flow is passed over the cylinder, a thermal plume like structure forms in a long upward direction, the plume size increases with increasing Reynolds number and/or decreasing the power-law index in both stream-wise and transverse directions, It is also appropriate to add here that in the case where the cylinder is having majority of the isotherm crowding hinting higher heat transfer rates. So far we have discussed only the effects of pure forced convection on flow field which is quite common in this paper. Still this is conducted to understand the effect of the superimposed thermal buoyancy.

The combined effects of thermal buoyancy and power law index can actually be established from Fig. 4, this figure shows representative results on streamlines and temperature contours (isotherm) at Reynolds number namely, $R e=40$, three values of the power-law index, and Richardson number $(0.1-0.3)$, in Fig. 4 it is observed that 
for all values of power-law indices with increase in the value of Richardson number, the separation point delays and moves towards the rear edge of the cylinder and accordingly, the wake region diminishes progressively both longitudinally and laterally. Eventually at some critical value of Richardson number there is no flow separation at all behind the obstacle, and above this critical value the streamlines are found to completely stick to the wall. Moreover, it can be also observed that increase in the value of power-law index requires more buoyancy strength up to reach the perfect suppressed flow. We can call this specific value of thermal buoyancy for which a separation flow is totally suppressed as the critical Richardson number (the maximum Richardson number required for a

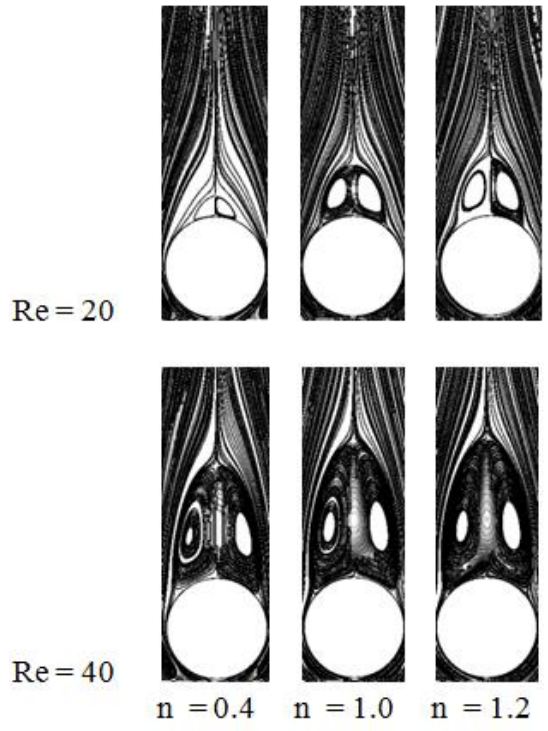

a
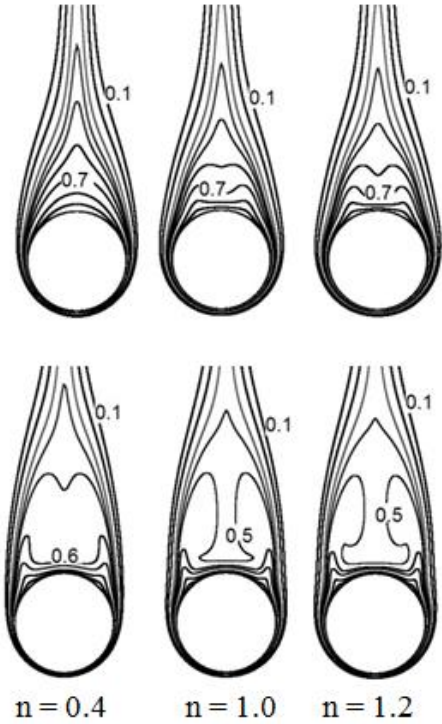

b

Fig. 3 Streamlines (a) and isotherms (b) around the cylinders for $R i=0, \operatorname{Pr}=50$ at different Reynolds numbers and power law indices

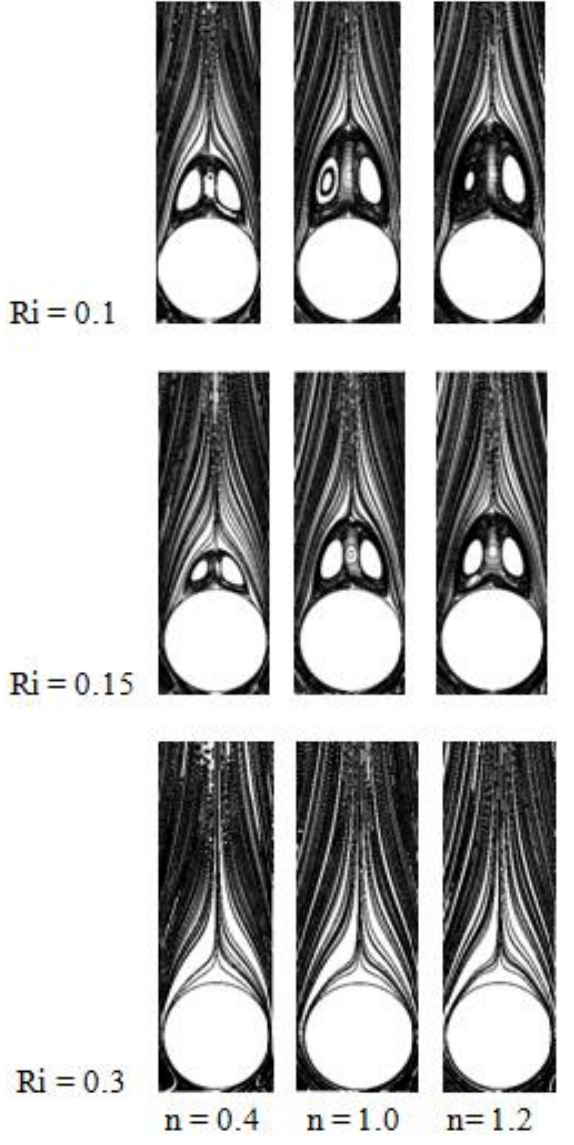

a
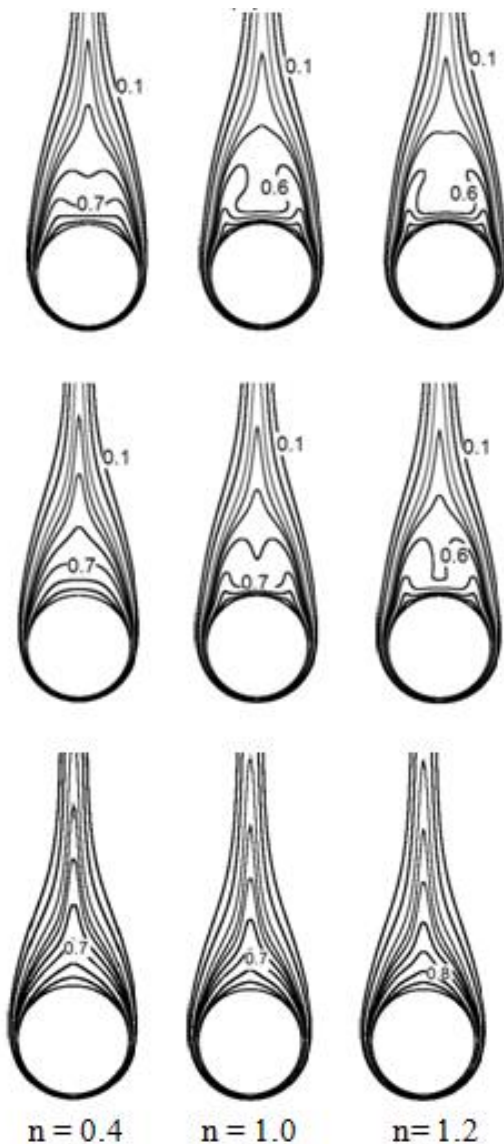

b

Fig. 4 Streamlines (a) and isotherms (b) around the cylinders for $R i=0.1-0.3$, for $\operatorname{Pr}=50$ at $\operatorname{Re}=40$ and power law indices 
complete suppression of flow separation). This flow behavior can be attributed by the fact that as the Richardson number increases (i.e., the thermal buoyancy effect becomes more pronounced) the velocity of the fluid particles behind the rear edge of the cylinder increases and moves toward the upward direction, so the inertia force is combined with the apparent viscous force, resulting in a separation delay and the point of separation moves down towards the rear edge of cylinder. Hence, it can be concluded that the effect of thermal buoyancy actually acts like a stabilizing force that reduces the hydrodynamic instability caused by increasing either $R e$ number or power-law index. This trend is consistent with that seen for Newtonian unconfined upward flow over square cylinder for moderate Reynolds number, the unsteady regime produces vortexes shedding in natural way when there is no superimposed thermal buoyancy. On the contrary the unsteady regime becomes extremely steady and there no vortexes shedding can be seen when the thermal buoyancy is added [18]. The isotherm contours shown in Fig. 4 reflect the physical phenomena seen from the analyses of streamlines patterns. Also, the longitudinal spread of temperature contours is seen to be reduced with increase in power-law index, on other hand, the lateral spread is reduced with increase in Richardson number (buoyancy strength).

Fig. 5 presents the variation of critical Richardson number (buoyancy strength) responsible for suppression of steady wake behind the object in vertical direction with Reynolds number for different value of power-law index $0.4-1.2$ for blockage ratio $(\beta=0.2)$. It can be observed that the maximum buoyancy strength requirement for flow suppression increases gradually with Reynolds number and a shear-thickening $(n>1)$ fluid requires more heating for suppression compared to its shear-thinning $(n<1)$ fluid counterpart. Since the flow instability increases with increasing Reynolds number or power-law index due to larger inertia and viscous effects, stronger buoyancy force is required to act against the hydrodynamic instability.

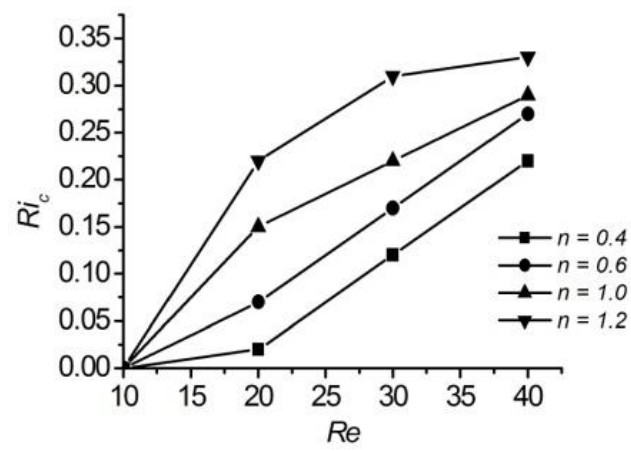

Fig. 5 Critical Richardson number as a function of Reynolds number for different power law index $(\operatorname{Pr}=50)$

The recirculation length $L^{*}$ is defined as the distance from the rear surface of the cylinder to the point of reattachment for the near closed streamline $(u=0$, $v=0$ ) on the line $x=0$ in the downstream section. This vital parameter can be used to quantify the phenomena of flow recirculation under the effect of thermal buoyancy. The variation of the non-dimensional recirculation length $L_{r}=\left(L_{r}^{*} / d\right)$ versus the Richardson number for different power power-law indices is presented in Fig. 6 at three different Reynolds: a $-R e=20 ; \mathrm{b}-\operatorname{Re}=30 ; \mathrm{c}-\operatorname{Re}=40$. For this particular blockage ratio $(\beta=0.2)$ the length of the recirculation zone increases with an increase in Reynolds number and / or power-law index for all values of Richardson. Also, it is clearly seen that at particular Reynolds number the recirculation length is significantly reduced with increase in the Richardson number for all power-law index, as explained earlier, the inertia force of the buoyancy acts like stabilizing factor which has a tendency to reduce the separation of the flow.

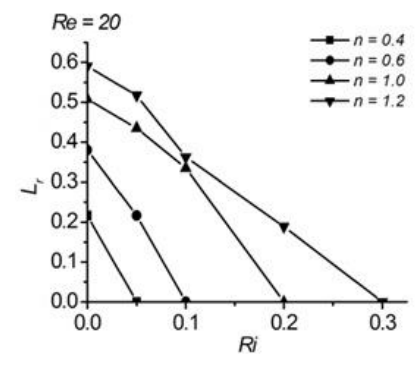

a

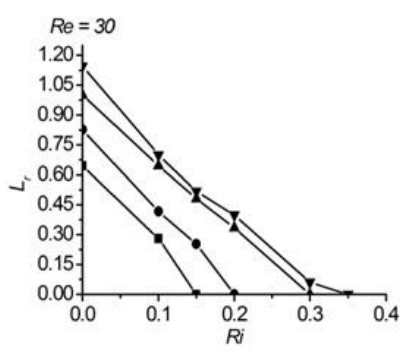

$\mathrm{b}$

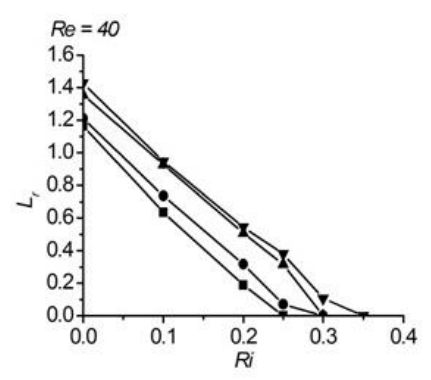

Fig. 6 Variation of dimensionless recirculation length with Richardson numbers for different power-law indices at different Reynolds numbers and $P r=50$.

In order to substantiate the fact of complete suppression of the wake behind the obstacle by adding of the superimposed thermal buoyancy. Fig. 7 is depicted at different representative Reynolds numbers, $R e=20-40$, to present the variation of skin friction coefficient at the rear edge of cylinder, $C_{f}$, with Richardson number for (a) $n=0.4$ and (b) $n=1$. The skin friction coefficient is defined as:

$$
C_{f}=\frac{\tau}{0.5 \rho V_{\max }^{2}},
$$

where $\tau$ is the local wall shear stress. The average value of shear stress is computed by surface averaging the integral local value along the respective small surface on the rear part of the cylinder. From graphs (a) and (b) It is observed that for an increase in the value of power-law index decreases the skin friction due to the rheological behavior of apparent viscosity behind the obstacle. Furthermore, at fixed power law index $n$, the friction coefficient reduces with increase in the Richardson number initially, reaches to a minimum at the critical Richardson number and then again increases at a very faster manner for all Re number. This is can be explained as follow: under the critical Richardson number the flow is still separated at the rear 
part of the cylinder, hence the skin friction at that side is small. A reserved trend is occurred above the critical Richardson number, the flow separation is completely disappeared resulting higher skin friction.

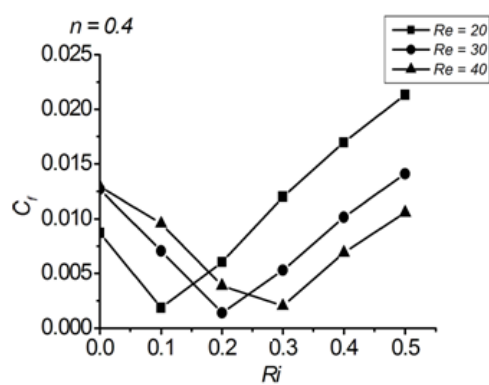

a

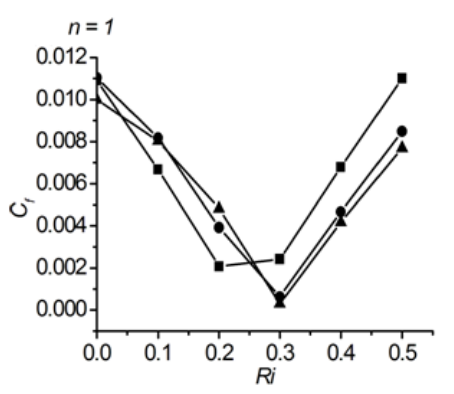

$\mathrm{b}$

Fig. 7 Skin friction coefficient at the rear edge as a function of Richardson number for $n=0.4,1.0$ for two Reynolds numbers and $\operatorname{Pr}=50$

The Fig. 8 shows the variation of the average Nusselt number with Richardson number for different values power-law index and Re at the fixed Prandtl number $P r=50$. The local Nusselt number is defined as:

$$
N u=\frac{h d}{k}=-\frac{\partial \theta}{\partial n_{s}},
$$

where $h$ and $n_{s}$ are the local surface heat transfer coefficient and the normal direction to the cylinder surface. The surface average heat transfer is obtained by surface averaging the integral local Nusselt number along the cylinder surface. Fig. 8 shows the average Nusselt number for four $R e$ number: a $-R e=10 ; \mathrm{b}-\operatorname{Re}=20$; c $-\operatorname{Re}=30$; $\mathrm{d}-R e=40$. From those graphs, the $\mathrm{Nu}$ number increases as usual with the Reynolds number for all cases. Also, at fixed $\mathrm{Re}$ a decrease in the value of power-law index promotes the rate of heat transfer due to lowering the apparent viscosity of the fluid near the cylinder and thus increasing the local Reynolds number and thinning the thermal boundary layer. Furthermore, in those graphs for all power law indices interesting variations are seen with respect to the Richardson number. Below the critical Richardson number, the heat transfer rate increases slightly, however, it increases with a faster rate above the critical buoyancy parameter due to higher contact between the fluid flow and the wall of cylinder. It can be concluded that generally using lower $n$ power-law index and/or increasing Richardson above the critical value can be economical to improve the efficiency of the process.

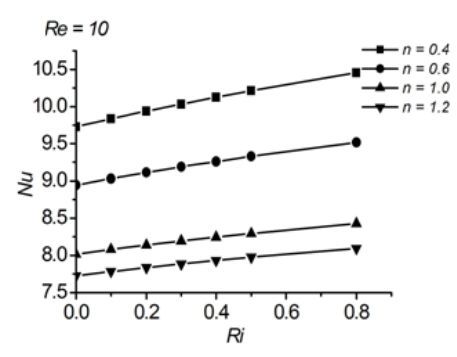

a

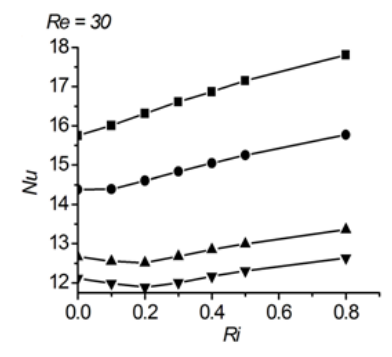

c

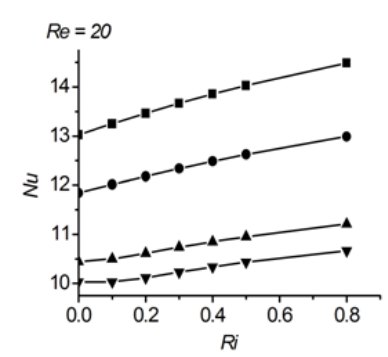

b

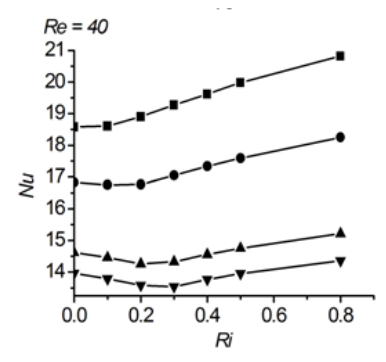

d
Fig. 8 Distribution of surface average Nusselt numbers with Richardson number for different Reynolds numbers and power-law indices for $\operatorname{Pr}=50$.

\section{Conclusions}

The combined free and forced convection of NonNewtonian power-law fluid flow and heat transfer around a confined heated circular cylinder is performed to examine the effect of buoyancy aided flow on the steady weak formed behind the obstacle for the confined channel $\beta=0.2$, upward flow of Non-Newtonian power-law for $n$ $0.4-1.2$ at low Reynolds number $\operatorname{Re} 10-40$, the critical Richardson numbers for the compete suppression of the wake are obtained for the cylinder in the aforesaid power law index and Reynolds number range. This is the most important contribution of the present work. Additionally, pertinent hydrodynamic parameters are obtained to further support the phenomena of wake suppression. In the absence of buoyancy effect, the recirculation length is found to increase with increasing the power-law index for this particular blockage ratio. On the contrary, the recirculation length is found to decrease with increasing strength of buoyancy and at critical value of Richardson number it disappears at all. The minimum value of skin friction coefficient can indicate the extreme critical Richardson number The Nusselt number increases with a decrease in the power-law index for all values of Richardson number. Moreover, the heat transfer rate increases more rapidly beyond the critical value of Richardson number.

\section{References}

1. Davis, D.V. 1983. Natural convection of air in a square cavity; a benchmark numerical solution, International Journal Number. Methods Fluids 3: 249-264.

http://dx.doi.org/10.1016/j.esd.2013.03.001.

2. Le Quéré, P.1991. Accurate solution to the square thermally driven cavity at high Rayleigh number, Computational Fluids 20: 29-41.

http://dx.doi.org/10.1016/j.esd.2013.03.001.

3. Dhinakaran, S.; Ponmozhi, J. 2011. Heat transfer 
from a permeable square cylinder to a flowing fluid, Energy Conversion and Management 52: 2170-2182. http://dx.doi.org/10.1016/j.enconman.2010.12.027.

4. Seyyedi, S.M.; Bararnia, H; Ganji, D.D.; GorjiBandpy, M.; Soheil Soleimani 2012. Numerical investigation of the effect of a splitter plate on forced convection in a two dimensional channel with an inclined square cylinder, International Journal of Thermal Sciences 61: 1-14. http://dx.doi.org/10.1016/j.ijthermalsci.2012.07.003.

5. Ribeiro, V.M.; Coelho, P.M.; Pinho, F.T.; Alves, M.A. 2014.Viscoelastic fluid flow past a confined cylinder: Three-dimensional effects and stability, Chemical Engineering Science 111: 364-380. http://dx.doi.org/10.1016/j.ces.2014.02.033.

6. Dhiman, A.K.; Chhabra, R.P.; Eswaran, V. 2008. Steady flow across a confined square cylinder: Effects of power-law index and blockage ratio, Journal NonNewtonian Fluid Mech 148: 141-150. http://dx.doi.org/10.1016/j.jnnfm.2007.04.010.

7. Koteswara Rao, P.; Sahu, Akhilesh K.; Chhabr, R.P. 2011. Momentum and heat transfer from a square cylinder in power-law fluids, International Journal of Heat and Mass Transfer 54: 390-403. http://dx.doi.org/10.1016/j.ijheatmasstransfer.2010.09.0 32.

8. Chandra, A.; Chhabra, R.P. 2011. Flow and forced convection heat transfer in Newtonian fluids from a semi-circular cylinder, International Journal of Heat and Mass Transfer 54: 225-241.

http://dx.doi.org/10.1016/j.icheatmasstransfer.2014.08. 025 .

9. Zeitoun, O.; Ali, Mohamed; Nuhait, A. 2011. Convective heat transfer around a triangular cylinder in an air cross flow, International Journal of Thermal Sciences 50: 1685-1697.

http://dx.doi.org/10.1016/j.ijthermalsci.2011.04.011.

10. Dipankar Chatterjee; Bittagopal Mondal 2012. Forced convection heat transfer from an equilateral triangular cylinder at low Reynolds numbers, Heat Mass Transfer 48: 1575-1587. http://dx.doi.org/10.1007/s00231-012-1006-x.

11. Greenblatt, D.; Wygnanski, I.J. 2000. The control of flow separation by periodic Excitation, Prog. Aerosp. Sci. 36: 487-545.

http://refhub.elsevier.com/S0017-9310(14)00041-6/h0 020 .

12. Gad-el-Hak, M. 2000. Flow Control: Passive, Active, and Reactive Flow Management, Cambridge University Press, $442 \mathrm{p}$.

http://refhub.elsevier.com/S0017-9310(14)00041-6/h0 015.

13. Dipankar Chatterjee; Bittagopal Mondal. 2014. Control of flow separation around bluff obstacles by superimposed thermal buoyancy, International Journal of Heat and Mass Transfer 72: 128-138.

http://dx.doi.org/10.1016/j.ijheatmasstransfer.2014.01.0 13.

14. Chhabra, R.P.; Richardson, J.F. 2008. NonNewtonian Flow and Applied Rheology, 2-nd ed, Butterworth-Heinemann, Oxford, 536 p.

15. Dipankar Chatterjee; Sakir Amiroudine 2010 Two- dimensional mixed convection heat transfer from confined tandem square cylinders in cross-flow at low Reynolds numbers, International Communications in Heat and Mass Transfer 37: 7-16.

http://dx.doi.org/10.1016/j.icheatmasstransfer.2009.10. 007.

16. Jaber Aboueian; Jahromi; Amin Behzadmehr 2011. Effects of inclination angle on the steady flow and heat transfer of power-law fluids around a heated inclined square cylinder in a plane channel, Journal of NonNewtonian Fluid Mechanics 166: 1406-1414. http://dx/doi.org/10.1016/j.jnnfm.2011.09.004.

17. Sivakumar, P.; Bharti, Ram Prakash; Chhabra, R.P. 2007. Steady flow of power-law fluids across an unconfined elliptical cylinder, Chemical Engineering Science 62: 1682 -1702. http://dx/doi.org/10.1016/j.ces.2006.11.055.

18. Chatterjee, D.; Mondal, B. 2012. Effect of thermal buoyancy on the two-dimensional upward flow and heat transfer around a square cylinder, Heat Transfer Eng. 33: 1-14.

http://refhub.elsevier.com/S0017-9310(14)00041-6/h0 125.

H. Laidoudi, M. Bouzit

S u m m a r y

This paper presents a comprehensive computational work on hydrodynamic and thermal phenomena of flow separation around a confined circular cylinder by superimposed thermal buoyancy. For that purpose, let us consider a confined flow of Non-Newtonian power-law fluid around a heated square cylinder in a twodimensional vertical channel. The effects of thermal buoyancy and power-Law index on the flow separation and the average Nusselt number are studied for the conditions: $(10 \leq R e \leq 40),(0.4 \leq n \leq 1.2),(0 \leq R i \leq 0.8), \operatorname{Pr}=50$ and blockage ratio $\beta=0.2$. The Reynolds numbers of flow are chosen in low range such that the flow remains steady and separated without imposition of the thermal buoyancy. In the steady flow regime the results show that the augmentation of the power-law index in the absence of thermal buoyancy causes a separation to grow. The thermal buoyancy delays the separation in different power-law indexes gradually and at some critical value of the buoyancy parameter it completely disappears resulting a stuck flow around a cylinder. Moreover, the recirculation length and skin friction are calculated to support the above finding. The decrease in the power-Law index increases the heat transfer rate. The Nusselt numbers are computed to predict the heat transfer rates of power-law fluids under the superimposed thermal buoyancy condition.

Keywords: Power-Law fluids, Steady flow, Thermal buoyancy, Flow suppression, Nusselt number Heat transfer, Critical Richardson number.

Received March 07, 2016

Accepted April 14, 2017 